\title{
Canagliflozin should be prescribed with caution to individuals with type 2 diabetes and high risk of amputation
}

\author{
Matilde Monteiro-Soares $^{1,2} \cdot$ Inês Ribeiro-Vaz ${ }^{1,3} \cdot$ Edward J. Boyko $^{4,5}$ (D) \\ Received: 19 February 2019 / Accepted: 8 March 2019/Published online: 2 April 2019 \\ (C) This is a U.S. government work and not under copyright protection in the U.S.; foreign copyright protection may apply 2019
}

Keywords Adverse drug reactions · Canagliflozin · Diabetic foot · Lower extremity amputation $\cdot$ SGLT2

\begin{tabular}{|c|c|}
\hline \multicolumn{2}{|c|}{ Abbreviations } \\
\hline CANVAS & $\begin{array}{l}\text { CANagliflozin cardioVascular } \\
\text { Assessment Study }\end{array}$ \\
\hline CANVAS-R & $\begin{array}{l}\text { CANagliflozin cardioVascular } \\
\text { Assessment Study-Renal }\end{array}$ \\
\hline DPP-4 & Dipeptidyl peptidase 4 \\
\hline FDA & Food and Drug Administration \\
\hline LEA & Lower extremity amputations \\
\hline SGLT2 & Sodium-glucose cotransporter 2 \\
\hline
\end{tabular}

\section{Introduction}

Around $80 \%$ of lower extremity amputations (LEA) in individuals with diabetes are preceded by a diabetic foot ulcer [1]. Diabetic foot ulcers usually occur due to a direct or indirect trauma associated with loss of protective sensation and are frequently accompanied by peripheral artery disease. Several factors increase the risk of LEA in diabetic individuals with or without diabetic foot ulcers, such as male sex, sensory neuropathy, peripheral artery disease, prior history of foot ulcer or amputation, poor glycaemic control, renal dysfunction, non-

Edward J. Boyko

eboyko@uw.edu

1 Center for Health Technology and Services Research (CINTESIS), Faculty of Medicine, University of Porto, Porto, Portugal

2 Department of Community Medicine, Information and Health Decision Sciences (MEDCIDS), Faculty of Medicine, University of Porto, Porto, Portugal

3 Porto Pharmacovigilance Center, Faculty of Medicine, University of Porto, Porto, Portugal

4 Veterans Affairs Puget Sound Health Care System (S-123-PCC), 1660 S. Columbian Way, Seattle, WA 98108, USA

5 University of Washington School of Medicine, Seattle, WA, USA
Asian ethnicity and presence of infection [2-8]. Recently, the Action to Control Cardiovascular Risk in Diabetes (ACCORD) trial, in which individuals were randomised to receive intensive therapy $\left(\mathrm{HbA}_{1 \mathrm{c}}<42 \mathrm{mmol} / \mathrm{mol}\right.$ $[<6 \%])$ or standard glycaemic control $\left(\mathrm{HbA}_{1 \mathrm{c}} 53-\right.$ $63 \mathrm{mmol} / \mathrm{mol}$ [7.0-7.9\%]), showed that the intensive regimen resulted in a significant decrease in LEA risk and that mean $\mathrm{HbA}_{1 \mathrm{c}}$ strongly predicted LEA occurrence [9]. One would, therefore, expect LEA risk to diminish with control of hyperglycaemia using glucose-lowering agents. Thus, the higher amputation risk with use of canagliflozin, a sodium-glucose cotransporter 2 (SGLT2) inhibitor effective in reducing blood glucose levels, reported by Matthews et al in this issue of Diabetologia [10], requires further scrutiny.

\section{The design of CANVAS and CANVAS-R and implications for clinical interpretation}

CANagliflozin cardioVascular Assessment Study (CANVAS) was begun in 2009 to demonstrate the cardiovascular safety of canagliflozin prior to registration [11]. CANagliflozin cardioVascular Assessment Study-Renal (CANVAS-R) was begun in 2014 to fulfil regulatory agency requirements post registration [12]. These studies were conducted in 30 countries in a total of 667 sites. CANVAS-R was designed to be completed simultaneously with CANVAS upon reaching a pre-specified endpoint number and follow-up duration.

It is important to note the restrictions placed on participant recruitment to understand to whom the results of these clinical trials apply. All participants had type 2 diabetes, with $\mathrm{HbA}_{1 \mathrm{c}}$ (as a measure of glycaemic control) between $53 \mathrm{mmol} / \mathrm{mol}$ and $91 \mathrm{mmol} / \mathrm{mol}(7.0 \%$ and $10.5 \%)$, and were either $\geq 30$ years with symptomatic atherosclerotic cardiovascular disease or $\geq 50$ years with diabetes duration $\geq 10$ years, systolic or treated hypertension, micro- or macro-albuminuria, or 
low HDL-cholesterol [10]. Therefore, whether the results of CANVAS and CANVAS-R, pertaining to both benefits and risks, apply to people not meeting these criteria, or those who would not agree to participate in a clinical trial, is unknown.

Assessment of major risk factors for nontraumatic amputation, such as prior amputation or history of peripheral vascular disease, was based on physician reporting [10], which we interpret to mean that no specific criteria were used or protocol followed for these measurements. We assume that this also applied to neuropathy assessment, another important risk factor for amputation. One cannot fault the investigators for not focusing on lower-limb risk factors since the association between canagliflozin and amputation was unanticipated. The lack of a systematic method for measurement of amputation risk factors is not likely to bias the results regarding amputation risk associated with treatment since participant randomisation would have likely equally allocated all risk factors, whether accurately measured or not.

With regard to capturing the amputation outcome, a plan was developed in July 2016 when a higher amputation risk was detected in the CANVAS Program, which included criteria and a procedure for recording amputation events, both prospectively and retrospectively [10]. It is unlikely that the detection of a higher amputation risk would have led to biased amputation assessment, either prospectively or retrospectively, due to the double-blind design of both CANVAS and CANVAS-R.

A total of 187 participants experienced an amputation during follow-up, with a total of 290 amputation events; 123 participants experienced a single amputation event. There were multiple amputations at varying locations on the same limb in $22 \%$ of individuals assigned to canagliflozin and $19 \%$ assigned to placebo [10], potentially reflecting failed initial amputation. The vast majority of amputations occurred while participants were using the randomised treatment $(75 \%)$ or within 30 days of its discontinuation (10\%). The majority of amputations were minor (below the ankle; 71\%), consistent with national US data, which demonstrates that the most common diabetes amputation level is below the foot; while, in people without diabetes, it is above the foot [13]. Similar elevated risks associated with canagliflozin were seen for both major (ankle or above) or minor (below the ankle) amputations [10]. No differences in amputation risk were noted at different canagliflozin dosages (300 mg vs $100 \mathrm{mg}$ ) or in CANVAS compared with CANVAS-R.

In the paper by Matthews and colleagues [10], a thorough investigation was performed to determine if the association between canagliflozin and amputation risk varied by the presence or absence of 25 factors. A standard method was used to assess such instances by insertion of interaction terms into the Cox regression model. There was no evidence for significant differences for known risk factors for amputation such as neuropathy, peripheral vascular disease, renal dysfunction, glycaemic control, or most of the other medications used to treat diabetes or associated conditions analysed. There was a single significant interaction $(p=0.03)$ between use of antithrombotic medication and canagliflozin, with amputation risk tending to be lower among canagliflozin users receiving these treatments than in canagliflozin users not receiving these treatments. However, the likelihood of one significant $p$ value at $p<0.05$ across 25 comparisons can be calculated as $1-(1$ $-0.05)^{25}=72 \%$. The Bonferroni correction for multiple comparisons, if applied, would have yielded an adjusted $p$ value for rejection of the null hypothesis of $0.05 / 25=0.002$. None of the tests of interaction in Fig. 3 in the publication by Matthews et al achieved a $p<0.002$ [10]. The non-standard and probably inaccurate measurements of peripheral vascular disease and neuropathy, however, may have limited the ability of the interaction analysis to identify whether these factors influenced canagliflozin-associated amputation risk due to potential misclassification of these conditions. The value of demonstrating a significant interaction would be to identify populations in whom canagliflozin treatment would be associated with a higher or lower risk of amputation, thereby facilitating clinical decision-making. The results of CANVAS/CANVAS$\mathrm{R}$ do not present convincing evidence of interaction.

Despite the negative results of the interaction analyses, it appears that more cases of amputation would be prevented by withholding canagliflozin treatment from people at higher risk for amputation than those at lower risk. As the overall risk elevation was about twofold, the absolute number of prevented cases would be greater in those with a higher baseline risk of amputation. Matthews et al demonstrate this in Table 3 in their paper [10] by estimating the number of amputations in subgroups that did not have independent risk factors for amputation identified in CANVAS and CANVAS-R, including male sex, non-Asian ethnicity, prior amputation, peripheral vascular (probably artery) disease, neuropathy, albuminuria and poorer glucose control. These risk factors for amputation are largely consistent with prior reports [2-8], as mentioned earlier in this commentary.

\section{Other research on the association between canagliflozin and amputation risk}

Both meta-analyses of RCTs and observational research have been conducted to further study the association between canagliflozin and amputation risk. Two meta-analyses, to our knowledge, have assessed this association [14, 15], but included only data from CANVAS and CANVAS-R [15] or $86 \%$ of participants in the analysis were derived from CANVAS and CANVAS-R [14]. This reflects the paucity of RCT data available on amputations and canagliflozin use from other clinical trials. 
Multiple observational studies have been conducted on the association between amputation and use of SGLT2 inhibitors, using databases from electronic health records or those maintained for administrative purposes. By our count, six studies have been published that used the Truven MarketScan health claims database from the USA, solely or in part, with varying results: three reported similar risk for amputation with canagliflozin compared with non-SGLT2-inhibitor glucoselowering agents [16-18]; two reported a lower amputation risk among those using SGLT2 inhibitors (where canagliflozin therapy comprised $70 \%$ of SGLT2 inhibitor use) compared with sulfonylurea agents, but not dipeptidyl peptidase 4 (DPP-4) inhibitors [19, 20]; and one reported a higher amputation risk for SGLT2 inhibitors compared with DPP-4 inhibitors, but not compared with sulfonylurea agents or non-metformin, non-SGLT2-inhibitor glucose-lowering agents [21]. Reconciling the different results reported from research using the Truven database appears to be needed, but is beyond the scope of this commentary.

Two published reports have addressed the subject of amputation in association with SGLT2 inhibitor use in other population databases. Udell et al reported on the association between SGLT2 inhibitors and amputation risk in the US Department of Defense Military Health System, which includes approximately 10 million enrolees [22]. A total of 79 amputations occurred and an increase in amputation risk was observed among users of any SGLT2 inhibitor, with the highest incidence of amputations in the canagliflozin-treated patients. However, no statistical comparison was conducted to assess this association. From the intention-to-treat data provided by Udell and colleagues (in supplemental Table 5 of their publication), there were 29 amputations among 7333 patients on canagliflozin who, during follow-up, contributed 15,046 person-years, and nine amputations in the equal number of propensity score-matched patients not receiving an SGLT-2 inhibitor who contributed 12,247 person-years. With these data, using Stata 15 (College Station, TX, USA) we calculated an incidence density ratio (IDR) of 2.63 (95\% CI 1.21, 6.30), a highly significant $(p=0.008)$ greater than twofold increase in amputation risk associated with canagliflozin. This result is adjusted for the covariates used to calculate the propensity score. Similar data were presented for empagliflozin and dapagliflozin, but the number of amputations was too small to conduct credible statistical analysis. We conclude from our secondary analysis that the observational results of Udell et al support a higher amputation risk associated with canagliflozin use. In addition, the nationwide health and administrative registers of Sweden and Denmark were also used to examine the association between SGLT2 inhibitors and amputation, finding a higher risk of amputation compared with glucagon-like peptide 1 (GLP-1) receptor agonists [23]. However, canagliflozin use comprised only $2 \%$ of prescriptions for SGLT2 inhibitors in only 324 patients. Hence, this research provides no information pertinent to the subject of amputation risk with this specific agent.

Adverse drug reaction reporting systems are another resource for identifying complications of pharmacotherapies. One such example is VigiBase, the World Health Organization (WHO) database of reported postcommercialisation adverse drug reactions [24]. We performed a search of VigiBase using the Medical Dictionary for Regulatory Activities (MedDRA) terminology for adverse drug reactions, with the preferred terms 'amputation', 'leg amputation' and 'toe amputation'. 'Amputation' was used to enhance sensitivity but we are aware that this term may capture upper-limb amputations and, for this reason, a second, more specific search was also performed using only the terms 'leg amputation' and 'toe amputation'. The database was accessed on 27 January 2019 and yielded 18,583 total adverse drug reaction reports related to canagliflozin, 10,553 reports related to dapagliflozin and 8427 reports related to empagliflozin. The search terms hits for canagliflozin greatly exceeded those for dapagliflozin and empagliflozin (Table 1). A review of the US Food and Drug Administration (FDA) Adverse Event Reporting System yielded similar results, with an amputation safety signal for canagliflozin but not dapagliflozin or empagliflozin [25].

These findings must be carefully interpreted as they refer to spontaneous adverse drug reaction reports and might be influenced by regulatory authorities' warnings from the US FDA or the European Medicines Agency, scientific presentations and publications, and the media. Moreover, these reports have no definitive causal relationship with drug exposure, being only suspected adverse reactions. It is also important to consider that the same case may include more than one term related with amputation, for example, amputation + leg amputation. Also, it is not possible to confirm medication exposure from such reports. Hence, adverse-event reporting medication databases represent weaker evidence than controlled observational research studies.

Table 1 Adverse drug reactions reported to VigiBase

\begin{tabular}{llll}
\hline ADR reported & \multicolumn{3}{l}{ Suspected drug } \\
\cline { 2 - 4 } & Canagliflozin & Empagliflozin & Dapagliflozin \\
\hline Amputation, $n(\%)$ & $163(0.88)$ & $5(0.06)$ & $2(0.02)$ \\
Leg amputation, $n(\%)$ & $322(1.73)$ & $13(0.15)$ & $3(0.03)$ \\
Toe amputation, $n(\%)$ & $885(4.76)$ & $33(0.39)$ & $23(0.22)$ \\
Total, $n$ & 18,583 & 8427 & 10,553 \\
\hline
\end{tabular}

ADR, adverse drug reaction 


\section{The roles of observational research and RCTs in clinical decision-making}

It is well understood that participants enrolled in an RCT usually are not widely representative of the general population, due to the need for inclusion and exclusion criteria designed to maximise trial validity and safety. Hence, observational research that includes participants not represented by clinical trial research may help to inform decisions about treatment efficacy and safety. In addition, observational research can provide information on relative safety and effectiveness of different diabetes treatments. CANVAS and CANVAS-R compared canagliflozin with placebo, but in the real world most patients with type 2 diabetes are treated with a glucoselowering agent that may, itself, alter risk of amputation.

We do not believe though that results from observational research that disagree with those of a well-conducted clinical trial negate the clinical trial. RCTs remain the gold standard for medical knowledge production. Even the best designed observational research, especially of a pharmaceutical agent, may be susceptible to error due to confounding by indication and other factors. The Women's Health Initiative (WHI) provides an excellent example of this in having conducted both an RCT and observational study of the association between menopausal hormone use and cardiovascular disease [26]. The results of these two study designs were discordant, with the RCT showing a higher incidence of coronary artery disease, stroke and venous thromboembolism with use of menopausal hormones, while the observational study showed a lower incidence of coronary artery disease and stroke with menopausal hormone use and no association with venous thromboembolism. This example demonstrates that, even with careful planning to identify and adjust for potential confounding, bias may not be minimised unless an impartial method, such as randomisation, is used to assign treatment.

\section{Potential mechanisms}

We propose here a theory regarding causal mechanisms for the higher amputation risk with canagliflozin treatment. This theory is built on interference by this pharmaceutical agent with the healing of diabetic foot ulcers; when unhealed, diabetic foot ulcers place the patient at higher risk of LEA. Thus, factors that impede healing may result in a higher risk of limb loss. Research has demonstrated roles for magnesium, calcium and vitamin D in promoting wound healing, with clinical trial evidence of enhanced diabetic foot ulcer healing with both magnesium and vitamin D supplementation [27-29]. Canagliflozin administration led to an increase of urine calcium excretion and magnesium wasting in animal models, and a decrease in $1,25(\mathrm{OH})_{2}$-vitamin $\mathrm{D}$ in hospitalised patients [30]. Further research is needed in humans to determine whether these effects on calcium, magnesium and vitamin D metabolism are specific to canagliflozin or a general characteristic of SGLT2 inhibitors.

\section{Conclusions}

CANVAS and CANVAS-R provide strong evidence that use of canagliflozin is associated with higher amputation risk, but also a lower risk of cardiovascular disease and renal disease progression. This does not appear to be an SGLT2 inhibitor class effect given the lack of elevated risk associated with empagliflozin in the EMPA-REG study [31] or the other pharmaceutical agents in this class, with the possible exception of ertugliflozin [32]. Therefore, the evidence is strong for favouring caution in canagliflozin use in patients with diabetes who are at risk for lower-limb amputation.

Funding VA Puget Sound Healthcare System provided support for EJB's participation in writing this commentary. The study sponsor was not involved in the design of the study; the collection, analysis, and interpretation of data; writing the report; or the decision to submit the report for publication.

Duality of interest EJB received support from Bayer AG for his participation at an expert committee meeting. MM-S and IR-V declare no duality of interest associated with this manuscript.

Contribution Statement All authors made substantial contributions to the conception and design, drafting the article, and giving final approval of the version to be published.

\section{References}

1. Pecoraro RE, Reiber GE, Burgess EM (1990) Pathways to diabetic limb amputation. Basis for prevention. Diabetes Care 13(5):513521. https://doi.org/10.2337/diacare.13.5.513

2. Boyko EJ, Seelig AD, Ahroni JH (2018) Limb- and person-level risk factors for lower-limb amputation in the prospective Seattle Diabetic Foot Study. Diabetes Care 41(4):891-898. https://doi. org/10.2337/dc17-2210

3. Hasan R, Firwana B, Elraiyah T et al (2016) A systematic review and meta-analysis of glycemic control for the prevention of diabetic foot syndrome. J Vasc Surg 63(2):22S-28S. https://doi.org/10. 1016/j.jvs.2015.10.005

4. Kim J-L, Shin JY, Roh S-G, Chang SC, Lee N-H (2017) Predictive laboratory findings of lower extremity amputation in diabetic patients: meta-analysis. Int J Low Extrem Wounds 16(4):260-268. https://doi.org/10.1177/1534734617737660

5. Shin JY, Roh SG, Sharaf B, Lee NH (2017) Risk of major limb amputation in diabetic foot ulcer and accompanying disease: a meta-analysis. J Plast Reconstr Aesthet Surg 70(12):1681-1688. https://doi.org/10.1016/j.bjps.2017.07.015

6. Tang ZQ, Chen HL, Zhao FF (2014) Gender differences of lower extremity amputation risk in patients with diabetic foot: a metaanalysis. Int J Low Extrem Wounds 13(3):197-204. https://doi. org $/ 10.1177 / 1534734614545872$

7. van Battum P, Schaper N, Prompers L et al (2011) Differences in minor amputation rate in diabetic foot disease throughout Europe 
are in part explained by differences in disease severity at presentation. Diabet Med 28(2):199-205. https://doi.org/10.1111/j.14645491.2010.03192.x

8. Zhou ZY, Liu YK, Chen HL, Yang HL, Liu F (2015) HbA1c and lower extremity amputation risk in patients with diabetes: a metaanalysis. Int J Low Extrem Wounds 14(2):168-177. https://doi.org/ 10.1177/1534734615593190

9. Goldman MP, Clark CJ, Craven TE et al (2018) Effect of intensive glycemic control on risk of lower extremity amputation. J Am Coll Surg 227(6):596-604. https://doi.org/10.1016/j.jamcollsurg.2018. 09.021

10. Matthews DR, Li Q, Perkovic Vet al (2019) Effects of canagliflozin on amputation risk in type 2 diabetes: the CANVAS Program. Diabetologia. https://doi.org/10.1007/s00125-019-4839-8

11. Neal B, Perkovic V, de Zeeuw D et al (2013) Rationale, design, and baseline characteristics of the Canagliflozin Cardiovascular Assessment Study (CANVAS) - a randomized placebo-controlled trial. Am Heart J 166(2):217-223 e11. https://doi.org/10.1016/j.ahj. 2013.05.007

12. Neal B, Perkovic V, Matthews DR et al (2017) Rationale, design and baseline characteristics of the CANagliflozin cardioVascular Assessment Study-Renal (CANVAS-R): a randomized, placebocontrolled trial. Diabetes Obes Metab 19(3):387-393. https://doi. org/10.1111/dom.12829

13. Geiss LS, Li Y, Hora I, Albright A, Rolka D, Gregg EW (2019) Resurgence of diabetes-related nontraumatic lower-extremity amputation in the young and middle-aged adult US population. Diabetes Care 42(1):50-54. https://doi.org/10.2337/dc18-1380

14. Li D, Yang JY, Wang T, Shen S, Tang H (2018) Risks of diabetic foot syndrome and amputation associated with sodium glucose cotransporter 2 inhibitors: a meta-analysis of randomized controlled trials. Diabetes Metab 44(5):410-414. https://doi.org/10.1016/j. diabet.2018.02.001

15. Radholm K, Wu JH, Wong MG et al (2018) Effects of sodiumglucose cotransporter-2 inhibitors on cardiovascular disease, death and safety outcomes in type 2 diabetes - a systematic review. Diabetes Res Clin Pract 140:118-128. https://doi.org/10.1016/j. diabres.2018.03.027

16. Adimadhyam S, Lee TA, Calip GS, Smith Marsh DE, Layden BT, Schumock GT (2018) Risk of amputations associated with SGLT2 inhibitors compared to DPP-4 inhibitors: a propensity-matched cohort study. Diabetes Obes Metab 20(12):2792-2799. https://doi. org/10.1111/dom.13459

17. Ryan PB, Buse JB, Schuemie MJ et al (2018) Comparative effectiveness of canagliflozin, SGLT2 inhibitors and non-SGLT2 inhibitors on the risk of hospitalization for heart failure and amputation in patients with type 2 diabetes mellitus: a real-world meta-analysis of 4 observational databases (OBSERVE-4D). Diabetes Obes Metab 20(11):2585-2597. https://doi.org/10.1111/dom.13424

18. Yuan Z, DeFalco FJ, Ryan PB et al (2018) Risk of lower extremity amputations in people with type 2 diabetes mellitus treated with sodium-glucose co-transporter-2 inhibitors in the USA: a retrospective cohort study. Diabetes Obes Metab 20(3):582-589. https://doi. org/10.1111/dom.13115

19. Chang HY, Singh S, Mansour O, Baksh S, Alexander GC (2018) Association between sodium-glucose cotransporter 2 inhibitors and lower extremity amputation among patients with type 2 diabetes. JAMA Intern Med 178(9):1190-1198. https://doi.org/10.1001/ jamainternmed.2018.3034

20. Dawwas GK, Smith SM, Park H (2019) Cardiovascular outcomes of sodium glucose cotransporter-2 inhibitors in patients with type 2 diabetes. Diabetes Obes Metab 21(1):28-36. https://doi.org/10. 1111/dom.13477

21. Yang JY, Wang T, Pate V et al (2019) Sodium-glucose cotransporter-2 inhibitor use and risk of lower-extremity amputation: evolving questions, evolving answers. Diabetes Obes Metab. https://doi.org/10.1111/dom.13647

22. Udell JA, Yuan Z, Rush T, Sicignano NM, Galitz M, Rosenthal N (2018) Cardiovascular outcomes and risks after initiation of a sodium glucose cotransporter 2 inhibitor: results from the EASEL population-based cohort study (evidence for cardiovascular outcomes with sodium glucose cotransporter 2 inhibitors in the real world). Circulation 137(14):1450-1459. https://doi.org/10.1161/ CIRCULATIONAHA.117.031227

23. Ueda P, Svanstrom H, Melbye M et al (2018) Sodium glucose cotransporter 2 inhibitors and risk of serious adverse events: nationwide register based cohort study. BMJ 363:k4365. https://doi.org/ 10.1136/bmj.k4365

24. Juhlin K, Karimi G, Ander M et al (2015) Using VigiBase to identify substandard medicines: detection capacity and key prerequisites. Drug Saf 38(4):373-382. https://doi.org/10.1007/s40264015-0271-2

25. Fadini GP, Avogaro A (2017) SGLT2 inhibitors and amputations in the US FDA Adverse Event Reporting System. Lancet Diabetes Endocrinol 5(9):680-681. https://doi.org/10.1016/S2213-8587(17) 30257-7

26. Prentice RL, Langer R, Stefanick ML et al (2005) Combined postmenopausal hormone therapy and cardiovascular disease: toward resolving the discrepancy between observational studies and the Women's Health Initiative clinical trial. Am J Epidemiol 162(5): 404-414. https://doi.org/10.1093/aje/kwi223

27. Oda Y, Tu CL, Menendez A, Nguyen T, Bikle DD (2016) Vitamin $\mathrm{D}$ and calcium regulation of epidermal wound healing. J Steroid Biochem Mol Biol 164:379-385. https://doi.org/10.1016/j.jsbmb. 2015.08.011

28. Razzaghi R, Pidar F, Momen-Heravi M, Bahmani F, Akbari H, Asemi Z (2018) Magnesium supplementation and the effects on wound healing and metabolic status in patients with diabetic foot ulcer: a randomized, double-blind, placebo-controlled trial. Biol Trace Elem Res 181(2):207-215. https://doi.org/10.1007/s12011017-1056-5

29. Razzaghi R, Pourbagheri H, Momen-Heravi M et al (2017) The effects of vitamin D supplementation on wound healing and metabolic status in patients with diabetic foot ulcer: a randomized, double-blind, placebo-controlled trial. J Diabetes Complicat 31(4): 766-772. https://doi.org/10.1016/j.jdiacomp.2016.06.017

30. Kalaitzoglou E, Fowlkes JL, Popescu I, Thrailkill KM (2019) Diabetes pharmacotherapy and effects on the musculoskeletal system. Diabetes Metab Res Rev 35(2):e3100. https://doi.org/10.1002/ dmrr. 3100

31. Inzucchi SE, Iliev H, Pfarr E, Zinman B (2018) Empagliflozin and assessment of lower-limb amputations in the EMPA-REG OUTCOME Trial. Diabetes Care 41(1):e4-e5. https://doi.org/10. 2337/dc17-1551

32. (2018) Ertugliflozin for type 2 diabetes. JAMA 319(23): 24342435. https://doi.org/10.1001/jama.2018.5840

Publisher's note Springer Nature remains neutral with regard to jurisdictional claims in published maps and institutional affiliations. 